\title{
Hole-spin initialization and relaxation times in InAs/GaAs quantum dots
}

\author{
F. Fras, B. Eble, P. Desfonds, F. Bernardot, C. Testelin, and M. Chamarro* \\ Institut des NanoSciences de Paris, UPMC Univ Paris 06, CNRS UMR 7588, 4 Place Jussieu, F-75252 Paris Cedex 05, France
}

A. Miard and A. Lemaître

Laboratoire de Photonique et Nanostructures, CNRS, Route de Nozay, F-91460 Marcoussis, France

(Received 4 February 2011; revised manuscript received 9 June 2011; published 12 September 2011)

\begin{abstract}
We study, at low temperature and zero magnetic field, the hole-spin dynamics in InAs/GaAs quantum dots. We measure the hole-spin relaxation time at a time scale longer than the dephasing time (about ten nanoseconds), imposed by the hole-nuclear hyperfine coupling. We use a pump-probe configuration and compare two experimental techniques based on differential absorption. The first one works in the time domain, and the second one is a new experimental method, the dark-bright time-scanning spectroscopy (DTS), working in the frequency domain. The measured hole-spin relaxation times, using these two techniques, are very similar, in the order of $T_{N}^{h} \approx 1 \mu \mathrm{s}$. It is mainly imposed by the inhomogeneous hole hyperfine coupling in the hole localization volume. The DTS technique allows us also to measure the hole-spin initialization time $\tau_{i}$. The hole spin is initialized by a periodic train of circularly polarized pulses at $76 \mathrm{MHz}$; we have observed that $\tau_{i}$ decreases as the power density increases, and we have measured a minimum value of $\tau_{i} \approx 100 \mathrm{~ns}$ in good agreement with a simple model [seeB. Eble, P. Desfonds, F. Fras, F. Bernardot, C. Testelin, M. Chamarro, A. Miard, and A. Lemaître, Phys. Rev. B 81, 045322 (2010)].
\end{abstract}

DOI: 10.1103/PhysRevB.84.125431

PACS number(s): 72.25.Rb, 72.25.Fe, 78.67.Hc, 78.55.Cr

\section{INTRODUCTION}

The spin of a carrier confined in a quantum dot (QD) is a good candidate for applications in nanoscale spintronics and as a solid-state qubit. ${ }^{1-3}$ A major obstacle in these applications is the decoherence due to the coupling of the spin with its environment. That is why the electron spin relaxation and decoherence sources in QDs have been intensively studied in the last few years; some more limited number of studies have centered on the hole spin. The main conclusions of these studies are that in moderate magnetic fields (1-10 T) and at low temperature, the electron $T_{1}^{e}$ and the hole $T_{1}^{h}$ spin-relaxation times are governed by the same mechanism, i.e., the spin-orbitmediated single-phonon scattering, ${ }^{4-8}$ which leads to relatively slow relaxation times in the range of milliseconds ${ }^{9-13}$ with $T_{1}^{h}$ five or ten times smaller than $T_{1}^{e} \cdot{ }^{12}$ However, the electron- and hole-spin coherence times $T_{2}^{e, h}$ have been found to be in the microsecond range up to $15 \mathrm{~K},{ }^{14-17}$ and for higher temperatures $T_{2}^{e}$ has shown a sharp decrease ${ }^{16}$ related to the modulation by phonons of the hyperfine (hf) interaction with the random fluctuating host nuclear spins.

The hf interaction has been identified as the dominant electron- and hole-spin dephasing source at weak or zero magnetic field and low temperature in QDs, ${ }^{18-25}$ leading to a dephasing time in the order of nanoseconds or tens of nanoseconds. Unlike electrons for which the contact term is the predominant contribution of the hf interaction, holes couple more weakly to nuclear spins via the anisotropic dipoledipole term of the $\mathrm{hf}$ interaction. ${ }^{24,25}$ Recently, the relative strength of the electron-nuclear coupling to the hole-nuclear coupling has been measured to be of the order of ten. ${ }^{26-28}$ Meanwhile, when the effect of the nuclear dipole-dipole interaction is neglected, ${ }^{29}$ theoretical works predict for both carriers two-time scales in the spin dynamics imposed by the hf interaction. ${ }^{18,20,30}$ The shorter time scale, in the nanosecond range, has been previously observed for both the electron and hole. ${ }^{14,22,23}$ Indeed, in a singly charged QD ensemble, each carrier-precession period is controlled by the hf field of the surrounding nuclei. At the carrier-precession timescale, due to the small variations of the nuclear effective field, it can be considered as frozen, but its dot-to-dot fluctuations induce a carrier spin dephasing and a partial relaxation of the ensemble spin carriers characterized by a dephasing time $T_{\Delta}^{e, h} \cdot{ }^{18,25}$ A longer relaxation time scale in the carrier spin dynamics also arises from the inhomogeneity of the carrier wave function, which makes the nuclear field change in time - the contribution of an individual nucleus to the nuclear field being proportional to the square of the carrier wave function at its place. Then, the slow carrier spin relaxation is related to this slow frozen field variation, which induces a carrier spin relaxation at a timescale $T_{N}^{e, h}$ longer than the ensemble dephasing time $T_{\Delta}^{e, h}\left(T_{N}^{e, h} \approx \sqrt{N} T_{\Delta}^{e, h}\right) .{ }^{18,31}$ The absence of the long time scale in the electron spin relaxation for an homogeneous hf coupling has been confirmed recently by Kozlov, ${ }^{32}$ who has calculated the exact solution of the hf hamiltonian describing the interaction of a single spin with nuclear spins and demonstrating that the incomplete spin relaxation is only fixed by the shorter time scale, i.e., the dephasing time $T_{\Delta}^{e}$.

In this paper, we address the hole-spin dynamics at low temperature and zero magnetic field at a scale longer than the dephasing time, $T_{\Delta}^{h}$, imposed by the hole hf interaction in $p$-doped InAs QDs (about ten nanoseconds). ${ }^{23}$ This slow scale has not yet been experimentally explored for hole spins in QDs. We introduce a new experimental method, working in frequency domain, to measure the hole-spin initialization and relaxation times: the dark-bright time-scanning spectroscopy (DTS). A pump-probe configuration is used to separate the long-living hole-spin polarization from the 
short-living photocreated trion-spin one. The intensity of the pump beam is switched on and off by an acousto-optic modulator, producing bright and dark excitation intervals of variable duration, with rise and fall times $\leqslant 50 \mathrm{~ns}$. Under these experimental conditions, the Fourier components of the hole-spin polarization signal are sensitive probes of the hole-spin initialization and relaxation times, in the range from $50 \mathrm{~ns}$ to $1 \mathrm{~ms}$.

\section{SAMPLE AND EXPERIMENTS}

The investigated sample contains $p$-doped InAs/GaAs QDs grown by molecular beam epitaxy on a (001) GaAs substrate; more details about this sample are given in Ref. 33. To probe the resident hole-spin polarization, we measured the photoinduced circular dichroism (PCD) in the QD sample. A permanent train of 2-ps pulses from a mode-locked Ti:sapphire laser with a 76- $\mathrm{MHz}$ repetition rate is split into pump and probe beams. The pump beam polarization is circular, and the probe beam is linearly polarized. After transmission through the sample, the probe beam is decomposed into its two circular components, and the difference in their intensities is measured with a balanced optical bridge to obtain the PCD signal. ${ }^{34,35}$ The probe beam is modulated at $470 \mathrm{~Hz}$, and the signal on the probe beam is recorded with a lock-in amplifier.

The spin initialization of the resident carriers in the $p$-doped QDs can be obtained by an optical pulsed excitation, circularly polarized and resonant with the trion transition. ${ }^{23,33}$ The efficient electron hf interaction imposes a coupling of both electron spin projections during the trion lifetime and, after emission of a circularly polarized photon, is at the origin of the hole-spin initialization. We will discuss in more detail the initialization mechanism in the last section of this article.

\section{A. Experiments in time domain using a pulse picker}

To explore the hole-spin dynamics at a long time scale, we have first increased the interval between the pump pulses by using a pulse picker in our experimental setup. ${ }^{36}$ The pump beam polarization is $\sigma+/ \sigma-$ modulated at $42 \mathrm{kHz}$ with a photoelastic modulator in order to prevent any dynamical polarization of the nuclear spins (DNP).

The inset of Fig. 1 shows the PCD decay curve obtained at $2 \mathrm{~K}$ when the period of the laser pulses is $\mathrm{N} \tau_{L} \approx 13 n s$ $(\mathrm{N}=1)$. Similar curves are obtained for each selected pump-pulse interval $N \tau_{L}$ with $\mathrm{N}=1,2,3, \ldots, 20$. The signal at negative delays, $\operatorname{PCD}\left(\mathrm{N} \tau_{L}\right)$, i.e., at a delay larger than the trion lifetime ( $T_{R} \approx 800 \mathrm{ps}$, see Ref. 23$)$, is unambiguously associated to the hole-spin polarization, and it is subject to the anisotropic hole hf interaction. In Fig. 1, we have plotted the normalized signal at negative delay, $\operatorname{PCD}(t=$ $\left.N \tau_{L}\right) / \mathrm{PCD}\left(t_{r e f}=1 n s\right)$ with $\mathrm{N}=2,3, \ldots, 20$, as a function of the time interval between the pump pulses. This experimental method presents, nevertheless, two main difficulties: first, the signal-to-noise ratio decreases as the interval between the pulses increases, because we must tune the peak powers of the pump and probe pulses to the same values for each laser period, and then we decrease the mean power; second, a simple analysis of the experimental decay curves assuming a mono-exponential decay is not pertinent in the case of a coupled dynamics of trion and hole spins imposed by a pulsed and periodic excitation. Indeed, the hole-spin polarization decreases not only due to the relaxation processes but also due to the reduction of signal as the interval between pump pulses increases. A model of this coupled and complex dynamics has, then, to be applied but implies the knowledge of several sample parameters. Solid, dashed, and dotted lines shown in Fig. 1 represent the theoretical dependencies given by the model described in Ref. 33, for different values of the hole-spin relaxation time. We underline here that in our previous works we have shown that the hole states in the studied sample have a mixed character. ${ }^{23}$ Experimental evidences of heavy-hole and light-hole mixing have been reported, before our works, and associated to the in-plane anisotropy and strain in InAs, CdSe, and CdTe self-assembled QDs (see Ref. 25-27 in Ref. 23). In the caption of Fig. $1, \beta$ is the hole-mixing coefficient defined in Refs. 23 and 25. We have also defined in Ref. 25 a hole-dephasing time independent of $\beta$ and denoted $T_{\Delta 0}$.

Finally, $T_{N}^{h}$ is determined to be close to $650 \mathrm{~ns} \pm 100 \mathrm{~ns}$, a much longer time than the $350 \mathrm{~ns}$ given by a rough analysis involving a simple exponential fit of the experimental data.

\section{B. Experiments in frequency domain: Dark-bright Time-scanning Spectroscopy (DTS)}

To measure the slow hole-spin dynamics at low temperature in InAs $p$-doped QDs, we introduce here a new experimental method, the DTS, based on differential absorption measurements and working in frequency domain. Until now, to measure a long spin relaxation time, authors have mainly adopted solutions based on the detection of the polarized photoluminescence excited by a probe beam, which has been delayed with respect to a previous pump beam. ${ }^{37-39}$ By

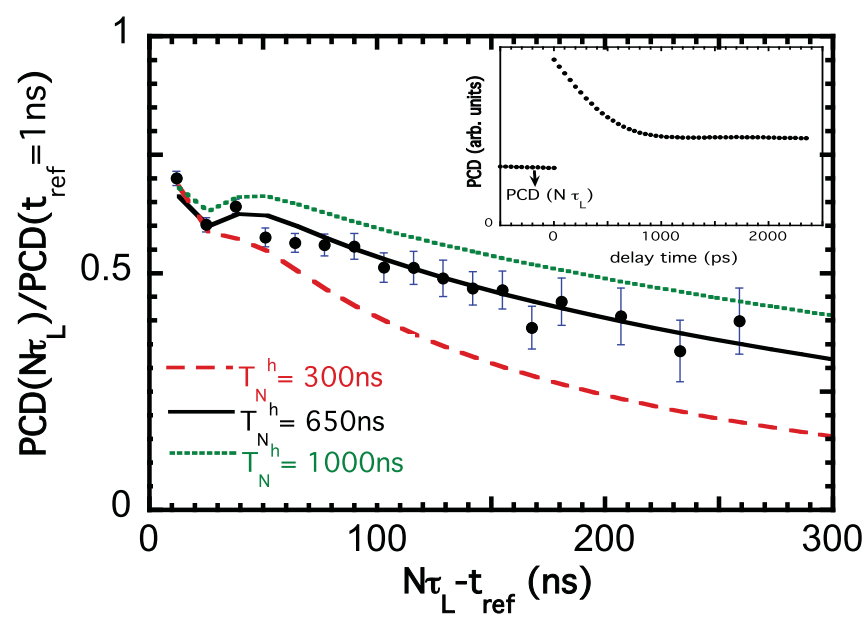

FIG. 1. (Color online) Circles represent the PCD signal at negative delays ( $N \tau_{L}$ with $\mathrm{N}=1,2,3 \ldots$ and $\left.\tau_{L}=13 n s\right)$, obtained by introducing a pulse picker after the laser source, as a function of the time interval between two pulses of the train. Solid, dashed, and dotted lines show the results of calculations for different values of $T_{N}^{h}$ obtained by using the dynamical model described in Ref. 33 and the following sample parameters: $T_{R}=800 \mathrm{ps}, T_{\Delta}^{e}=500 \mathrm{ps}, \beta=0.45$, $T_{\Delta 0}^{h}=5 n s$, the Landé electron g-factor $g_{e}=-0.4$, and the Landé hole g-factor $g_{h}=1.5$. Inset: PCD signal as function of pump-probe delay, obtained at $\mathrm{B}=0 \mathrm{~T}$ and $2 \mathrm{~K}$ after a pulsed excitation at $1.32 \mathrm{eV}$. 
changing the time delay between the pump and probe beams, a signature of the spin polarization in the time domain is obtained. In this configuration, the intensity of excitation is modulated by an acousto-optic modulator in order to make a difference between the pump and probe pulses through the variation of the pulse temporal width, rather than the pulse power intensity (which is the common way in the two-beam pump-probe configuration).

The DTS technique is based on a differential absorption measurement and uses a two-beam pump-probe configuration, measuring the PCD and allowing the separation of the longliving hole-spin polarization from the short-living contribution of the photocreated (positively charged) trion. The intensity of the pump beam is modulated by an acousto-optic modulator, which produces bright and dark excitation intervals of variable duration, with rise and fall times $<50 \mathrm{~ns}$ [see Fig. 2(a)], and then produces a periodic excitation. The analyzed PCD(13-ns) signal, which is proportional to the hole-spin polarization, is modulated at the acousto-optic modulation frequency $f_{m}$ [see Fig. 2(c)] and is also a periodic function $\mathrm{F}(\mathrm{t})$ with period $\mathrm{T}_{\mathrm{m}}=1 / f_{m}$.

If we assume an exponential rise with initialization time $\tau_{i}$ when the pump is "on" and an exponential relaxation with characteristic time $T_{N}^{h}$ when the pump is "off," $\mathrm{F}(\mathrm{t})$ can be written as follows:

$$
\left.\begin{array}{rl}
F(t) & =A\left[1-\eta e^{\frac{-t}{\tau_{i}}}\right], \quad 0<t<T_{b} \\
F(t) & =A\left[1-\eta e^{\frac{-T_{b}}{\tau_{i}}}\right] e^{-\left(\frac{t-T_{b}}{T_{N}^{h}}\right)}, \quad T_{b}<t<T_{m}
\end{array}\right\}
$$

where $T_{b}$ and $T_{d}$ are the bright and dark durations, respectively.

We measure the Fourier components of the $\operatorname{PCD}(13 \mathrm{~ns})$ signal as a function of acousto-optic modulation frequency $f_{m}$. As already said, the probe beam is also modulated at low frequency. In order to improve the signal-to-noise ratio, the signal on the probe beam is recorded with a double stage of lock-in amplifiers. We first extract the signal modulated at high frequency, $f_{m}$, and this signal is then sent to two

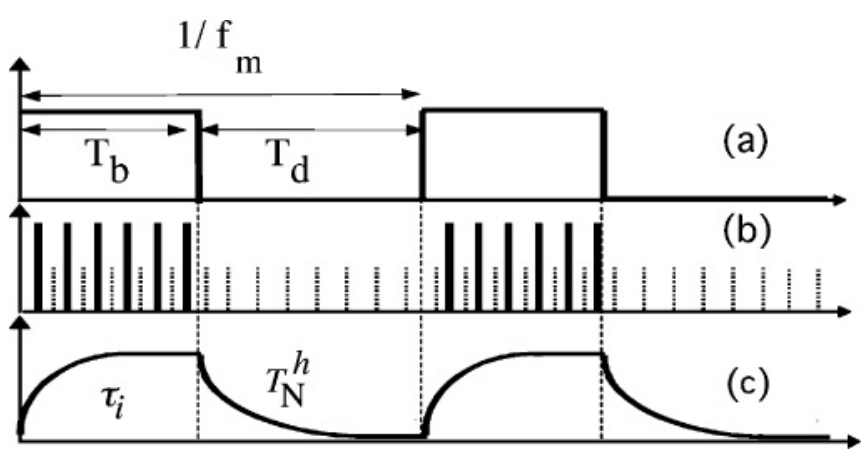

FIG. 2. Scheme giving the principle of the DTS technique used to determine the hole-spin polarization and relaxation times of InAs QDs. (a) Intensity modulation function imposed by the acousto-optic modulator to the pump beam. (b) Pump (thick lines) and probe (thin lines) pulses as function of time. (c) Temporal dependence of the modelized PCD signal. similar lock-in amplifiers, each of them extracts separately its quadratures modulated at $470 \mathrm{~Hz}$. Figure 3 shows results obtained in $p$-doped InAs QDs by applying the DTS to the PCD(13 ns) signal. The module and phase of the measured Fourier components are displayed for the first (a), second (b), and third (c) harmonics of the $\operatorname{PCD}(13 \mathrm{~ns})$ signal. The bright interval $T_{b}=10 \mu \mathrm{s}$ is kept constant, while we scan the dark interval $T_{d}$ from $190 \mu$ s to $100 \mathrm{~ns}\left(f_{m}\right.$ is then scanned from $5 \mathrm{kHz}$ to $99 \mathrm{kHz}$ ). Similar results are obtained by setting constant the dark interval and scanning the bright interval or by setting a constant $50 \%$ duty and scanning the frequency $f_{m}$. The module and phase of the measured harmonics are fitted simultaneously to the Fourier components of the periodic function given in Eq. (1), using only two fitting parameters, $\tau_{i}$ and $T_{N}^{h}$; in Fig. 3, solid lines represent the best fit to the experimental data. For comparison, Figs. 3(a)-3(c) also show the calculated dependences on $f_{m}$ of the Fourier components for the following two cases: dashed lines (dashed lines with circles) correspond to $\tau_{i}=0$ and $T_{N}^{h}=0\left(T_{N}^{h} \neq 0\right.$ and $\left.\tau_{i}=0\right)$. For the module, a logarithmic scale was used to underline the sensitivity of the fitting to the parameters. We observe that the maxima and minima of the module, as well as the singularities of the phase of each harmonic, are satisfactorily reproduced by the fitting curves. The higher the harmonic is, the better the precision on the determination of $\tau_{i}$ and $T_{N}^{h}$ (but the weaker the associated experimental signal). We obtain a good compromise by choosing to analyze the second harmonic; at $2 \mathrm{~K}$ and $\mathrm{B}=0 \mathrm{~T}, T_{N}^{h}$ is determined to be equal to $900 \mathrm{~ns} \pm 100 \mathrm{~ns}$. In the DTS configuration, the pump beam has a fixed helicity; we underline, however, that the measured $T_{N}^{h}$ is not very sensitive to the modulation regime (fixed bright time, fixed dark time, or fixed $50 \%$ duty), and we also underline that the $T_{N}^{h}$ value is very similar to the value obtained in Sec. IIA), i.e., for a high-frequency modulation of the pump helicity and in the absence of any DNP. That is why we exclude the presence of a strong DNP in the DTS configuration.

We note that the proposed experimental method could be extended to the measurement of initialization and relaxation times of other origins and magnitudes, in the range from $50 \mathrm{~ns}$ to $1 \mathrm{~ms}$. The lower limit of this range is fixed by the rising time of the acousto-optic modulator used to modulate the pump beam intensity; the upper limit is fixed, for a single modelocking analysis of the detected signal at $f_{m}$, due to the fact that dark and bright times have to be scanned in such a way as to be much larger than the measured time and compatible also with a good signal-to-noise mode-locking detection $\left(f_{m} \geqslant\right.$ $100 \mathrm{~Hz})$.

\section{DISCUSSION}

There are very few theoretical studies of hole-spin relaxation in QDs at low or zero magnetic field, ${ }^{40,41}$ but it appears that, contrary to the high magnetic field regime, the two-phonon spin-orbit-mediated process becomes the dominant mechanism instead of the single-phonon one; the predicted hole-spin relaxation time is in the order of several milliseconds ${ }^{41}$ for zero magnetic field. That is why we assume that the mechanism at the origin of the measured slow hole-spin relaxation, with characteristic time $T_{N}^{h}$, is the hole $\mathrm{hf}$ coupling acting at a longer time scale than the hole-spin 

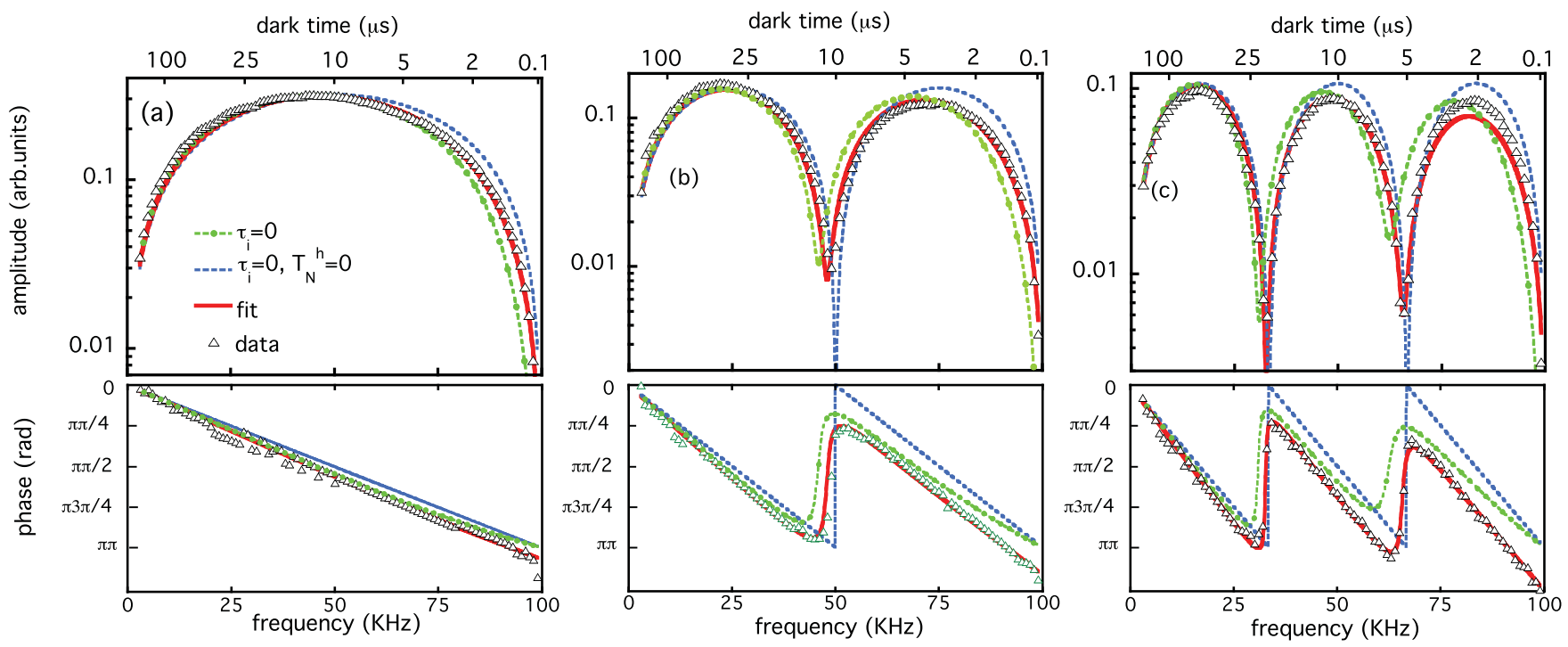

FIG. 3. (Color online) Upper (lower) panel: the module (phase) of the Fourier components are shown as function of modulation frequency, $f_{m}$, and dark time $T_{d}:$ (a) first harmonic, (b) second harmonic, and (c) third harmonic. Triangles represent experimental values. Lines represent theoretical curves with $\tau_{i}=T_{N}^{h}=0$ [dashed line (blue)] and $\tau_{i}=0$ and $T_{N}^{h} \neq 0$ [dashed line with circles (green): (a) $T_{N}^{h}=890 n s,(b)$ $T_{N}^{h}=995 \mathrm{~ns}$, (c) $\left.T_{N}^{h}=845 \mathrm{~ns}\right]$; red solid lines are fits of the experimental data with the following parameters: (a) $\tau_{i}=380 \mathrm{~ns}, T_{N}^{h}=890 \mathrm{~ns}$; (b) $\tau_{i}=490 \mathrm{~ns}, T_{N}^{h}=995 \mathrm{~ns}$; (c) $\tau_{i}=600 \mathrm{~ns}, T_{N}^{h}=845 \mathrm{~ns}$. The experimental data in (a), (b), and (c) have been obtained at low excitation densities and for slightly different excitation conditions; hence, $\tau_{i}$ varies from one curve to another

dephasing time $T_{\Delta}^{h}$ (about ten nanoseconds) and is related to inhomogeneities of the carrier wave function. We note that in the DTS technique we measure the $\operatorname{PCD}(13 \mathrm{~ns})$, i.e., at a fixed time that is comparable to, or larger than, the hole-dephasing time $T_{\Delta}^{h}$, which is why DTS measurements give information, essentially, on the slow hole-spin dynamics, and they are very few affected by the fast initial hole-spin decay. Concerning slow dynamics, theoretical papers predict a nonexponential spin relaxation; ${ }^{7,31,42}$ here the measured Fourier components are analyzed assuming an exponential decay, and in this sense our experiments give a characteristic time but do not give details of the complex relaxation of the hole spin.

The time $T_{N}^{h} \approx T_{\Delta 0}^{h} \sqrt{N} \approx \hbar N / C$ ( $C$ is the hole hf constant, see Ref. 25 for the $C$ and $T_{\Delta 0}^{h}$ definitions) and has to be compared to the electron spin time at the same scale, $T_{N}^{e} \approx \hbar N / A$ with $A$ the electron hf constant. ${ }^{18}$ At low temperature and in the absence of an applied magnetic field, Cheng et al. ${ }^{11}$ have obtained in interface-fluctuation GaAs QDs $T_{N}^{e}=34 \mu s$, and Pal et al. ${ }^{43}$ in InP QDs have measured $T_{N}^{e}=1 \mu s$. These values compare well with our experimental determination of $T_{N}^{h}$ because recent studies have shown that $\mathrm{C}$ is about ten times smaller than $A,{ }^{23,26-28}$ but the interface-fluctuation GaAs and InP QDs are larger than the InAs QDs studied in this work (N is larger).

The value of the initialization time $\tau_{i}$ depends on the conditions of the initialization process. The initialization of a single electron spin has been demonstrated under application of a magnetic field in Voigt ${ }^{44,45}$ or Faraday ${ }^{46}$ geometry and cw excitation. The initialization in the Voigt configuration is three orders of magnitude quicker than in the Faraday configuration, but the fidelity of this latter configuration is greater. Recently, in absence of an applied magnetic field, Ramsay et al. ${ }^{47}$ have demonstrated the sequential hole-spin initialization by the ionization of a spin-polarized electron-hole pair with an $81 \%$ fidelity, and Gerardot et al ${ }^{13}$ have also obtained a high fidelity of the spin state initialization for a single hole under $\mathrm{cw}$ optical excitation. In the past, Shabaev et al. ${ }^{48}$ have proposed the initialization of a single electron spin placed in a transverse magnetic field by a combination of circularly polarized optical $\pi$ pulses, and the authors have predicted a spin polarization of 99\% after eight pulses.

In our experiments, we have measured the spin initialization time of the hole spin in an ensemble of QDs under a periodic pulsed excitation in the absence of an applied magnetic field. Figure 4(a) shows a scheme of the hole-spin polarization mechanism. Figure 4(b) shows our measured $\tau_{i}$ as function of mean excitation power density, pulsed at $76 \mathrm{MHz} .^{49}$

By using the model given in Ref. 33, we are also able to calculate the hole-spin polarization level at negative time delay, $\rho_{h}=\frac{2}{3} J_{z}\left(n \tau_{L}\right)$ (where $n$ is an integer number, and $J_{z}$ denotes the component of the hole spin $\vec{J}$ along $\mathrm{z}$ ), versus number $\mathrm{n}$ of pump pulses [see inset of Fig. 4(b)]. This time dependence is obtained by considering the dynamic equations of the electron and hole spins, $\vec{S}$ and $\vec{J}$, respectively:

$$
\frac{d \vec{S}(t)}{d t}=\frac{g_{e} \mu_{B}}{\hbar} \vec{B}_{N}^{e} \times \vec{S}(t)-\frac{\vec{S}(t)}{T_{R}}
$$

and

$$
\frac{d \vec{J}(t)}{d t}=\frac{g_{h} \mu_{B}}{\hbar} \vec{B}_{N}^{h} \times \vec{J}(t)+3 \frac{(\vec{S} \cdot \vec{z}) \vec{z}}{T_{R}}-\frac{\vec{J}(t)}{T_{N}^{h}},
$$

where $g_{e}$ and $g_{h}$ are the Landé factors of the electron and hole. $\vec{B}_{N}^{e}$ and $\vec{B}_{N}^{h}$ are the effective nuclear fields induced by hf coupling and acting on the electron and hole, respectively. $\mu_{B}$ is the Bohr magneton. Without pumping, the system is at the thermodynamic equilibrium, and there is no spin: 

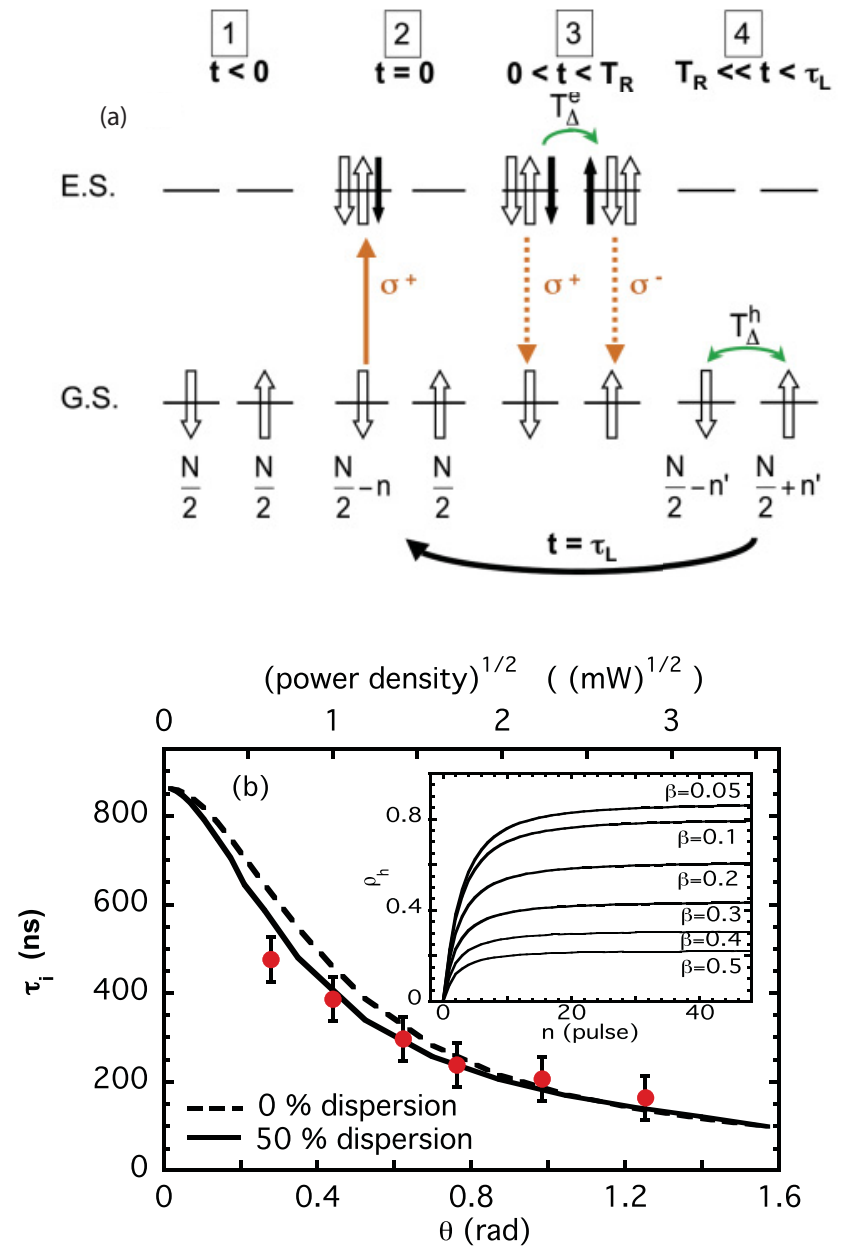

FIG. 4. (Color online) (a) 1 - $t<0$ : The ensemble of studied singly charged InAs QDs is initially unpolarized: $\frac{N}{2}$ of them contain resident spin-down heavy holes $\left(J_{z}=-\frac{3}{2}\right)$ and $\frac{N}{2}$ contain spin-up heavy holes $\left(J_{z}=+\frac{3}{2}\right)$. Hole spins are indicated by white arrows. [GS: ground state (hole); ES: excited state (trion)]. $2-\mathrm{t}=0: \mathrm{A} \sigma^{+}$pump pulse resonantly excites $n$ QDs containing spin-down holes to the $S_{z}=-\frac{1}{2}$ trion state. According to the selection rules, the angular momentum of these QDs has been increased by one unit. In the $-\frac{1}{2}$ trion state, the downward black arrow represents a spin-down electron. $3-0<\mathrm{t}<$ $T_{R}$ : After the excitation pulse, the ESs and the GSs are spin polarized. During the trion lifetime $T_{R}$, the electron-nuclear interaction, characterized by the ensemble dephasing time $T_{\Delta}^{e}$, coherently couples the two electron spins $\uparrow$ and $\downarrow$ (with spin components $S_{z}=+1 / 2$ or $-1 / 2$, respectively), within each excited QD. $4-\mathrm{T}_{\mathrm{R}} \ll \mathrm{t}<\tau_{\mathrm{L}}$ : The spontaneous decay of the trion states by emission of polarized photons (dashed vertical arrows in scheme 3 ) has led to an efficient resident hole-spin cooling in their GS: $\frac{N}{2}-n^{\prime}$ QDs have a spin-down hole, while $\frac{N}{2}+n^{\prime}$ have a spin-up hole. The hole-spin dynamics, before the next excitation, is then determined by the hole-nuclear interaction, characterized by the ensemble dephasing time $T_{\Delta}^{h}$. (b) $\tau_{i}$ as a function of the square root of the density of excitation. Circles correspond to the experimental data, obtained under the same excitation conditions. Lines are two fits with the model described in Ref. 33, assuming an inhomogeneity of Rabi angle values of $50 \%$ (solid line) or without inhomogeneity (dotted line), and taking the same sample parameters as used in Fig. 1. The only fitting parameter is the coefficient of proportionality between values of the square root of power density and values of $\theta$. Inset: $\rho_{h}$ versus number of pump $\pi-$ pulses, for different heavy-light hole-mixing parameters $\beta$.
$\vec{S}(t<0)=\vec{J}(t<0)=\overrightarrow{0}$ [see Fig. 4(a)]. Then, at $\mathrm{t}=0$, a train of $\sigma^{+}$polarized pulses is applied. The spin dynamics is calculated by averaging on the nuclear configurations (cf. Ref. 33) and by using the Eqs. (2) and (3) with the condition $S_{z}\left(n \tau_{L}^{+}\right)=\frac{1}{4} \sin ^{2} \frac{\theta}{2}\left(\frac{2}{3} J_{z}\left(n \tau_{L}^{-}\right)-1\right)$ for every $n^{\text {th }}$ pulse. $\theta$ is the Rabi angle associated to the pump pulse, and $\tau_{L}^{+}\left(\tau_{L}^{-}\right)$ corresponds to the time just after (before) pump pulse arrival. Curves in the inset of Fig. 4(b) are obtained for $\theta=\pi$. We observe that the hole-spin polarization level at negative delay, $\rho_{h}$, increases as $\mathrm{n}$ increases until a saturation value $\rho_{\max }$. We have defined the calculated initialization time $\tau_{i}$ as the time needed to obtain a value of the hole-spin polarization equal to (1-1/e) of the saturation value $\rho_{\max }$.

For $n$-doped QDs, a clear Rabi regime was previously observed. ${ }^{3,50}$ Despite comparable experimental conditions, we have not observed a Rabi regime in our $p$-doped QDs. This may be due to the presence of a larger inhomogeneity in the Rabi angle, because of the QD dipole dispersion in the optical pump spot. For comparison with experimental results, we have considered a Gaussian distribution with a mean Rabi angle $\theta=A \sqrt{I_{p}}: I_{p}$ is the pump power, $A$ is the single fitting parameter, and all the other parameter values used in the model have been obtained from previous studies. ${ }^{33}$ Solid and dotted lines in Fig. 4(b) show the calculated $\tau_{i}$ as function of Rabi angle $\theta$. We note that when $\theta \rightarrow 0$, $\tau_{i} \rightarrow T_{N}^{h}$ (but at the same time $\rho_{\max } \rightarrow 0$ ). We observe a good agreement between the experimental and theoretical $\tau_{i}$ dependences. For an estimated average Rabi angle $\theta \approx \pi / h$, we measured $\tau_{i} \approx 100 n s$ (eight pulses). The inset of Fig. 4(b) shows that the value of $\rho_{\max }$ obtained for a $\pi$-pulse depends on the heavy-light mixing character of the hole states in the QDs, characterized by the parameter $\beta$ (see Refs. 23 and 25). We underline that the number of pulses needed to initialize the hole-spin polarization is nearly constant and varies from four to three when $\beta$ varies from 0.5 to 0.05 , meanwhile the value of $\rho_{\max }$ varies from $20 \%$ to $90 \%$. Hence $\tau_{i}$ is mainly imposed by the electron-nuclear coupling strength and the trion lifetime and does not change significantly with $\beta$. This can be explained by the efficiency of the polarization mechanism associating optical pumping, electron-nuclear hf evolution, and trion recombination. If the spin-down $(\Downarrow)$ hole density is $\rho_{\Downarrow}$ before excitation by a $\sigma^{+}$polarized pulse, one can show, in the framework of Ref. 18, that after excitation and trion recombination, part of the spin-down holes have flipped. This quantity, $\beta$-independent, is equal to $\sin ^{2} \frac{\theta}{2} G(x) \rho_{\Downarrow}$ with $x=\frac{T_{\Delta}^{e}}{T_{R}}$ and $G(x)=\frac{1}{3}\left[1-2 x^{2}\left(1-\sqrt{\pi} x e^{x^{2}} \operatorname{erfc} c(x)\right)\right]$, if $T_{N}^{h} \gg T_{\Delta}^{e}$. For $T_{\Delta}^{e}=500 \mathrm{ps}, \theta=\pi$, and $T_{R}=800 \mathrm{ps}, 23 \%$ of the initial spin-down states are in the opposite spin state after trion recombination, so that only three or four pulses are needed to flip the spin-down holes in a ratio close to (1-1/e). Finally, one can note that the hole-spin relaxation is slower for more pure heavy holes and that an improved hole-spin initialization could be accomplished for highly pure heavy holes.

\section{CONCLUSION}

To conclude, the hole-spin dynamics in p-doped InAs/GaAs QDs was studied at low temperature and in the 
absence of an applied magnetic field. We have shown, by using the DTS technique, that the Fourier components of the modulated hole-spin polarization signal are sensitive probes of the initialization and relaxation times.

The measured slow hole-spin relaxation time, $T_{N}^{h} \approx 1 \mu \mathrm{s}$, is mainly associated to the slow time variation of the frozen nuclear effective field acting on the hole spin and related to the inhomogeneous profile of the hole wave function. We have also shown that the fidelity of the hole-spin initialization obtained by a periodic pulsed excitation is maximal for pure heavy-hole states. The initialization time $\tau_{i}$ is imposed by the electronnuclear hf coupling and it is fixed at about a few tens of nanoseconds. At a very low density of excitation, $\tau_{i}$ takes a maximum value and becomes equal to $T_{N}^{h}$. Experiments are in good agreement with our model and show that $\tau_{i}$ decreases as the density of excitation increases.

Finally, because we have shown that the DTS provides detailed and valuable clues to the hole-spin initialization and relaxation times, this technique could be extended to the measurement of the initialization and relaxation times of other magnitudes, in the range from $50 \mathrm{~ns}$ to $1 \mathrm{~ms}$.

\section{ACKNOWLEDGMENTS}

This work is supported by the QUAMOS Project No. ANR09-NANO-030 funded by the French National Agency (ANR). *maria.chamarro@insp.jussieu.fr

${ }^{1}$ D. Loss and D. P. DiVincenzo, Phys. Rev. A 57, 120 (1998).

${ }^{2}$ D. D. Awschalom, D. Loss, and N. Samarth (eds), Semiconductor Spintronics and Quantum Computation (Springer, New York, 2002).

${ }^{3}$ M. Chamarro, F. Bernardot, and C. Testelin, J. Phys.: Condens. Matter 19, 445007 (2007).

${ }^{4}$ A. V. Khaetskii and Y. V. Nazarov, Phys. Rev. B 61, 12639 (2000); 64, 125316 (2001).

${ }^{5}$ L. M. Woods, T. L. Reinecke, and Y. Lyanda-Geller, Phys. Rev. B 66, 161318(R) (2002).

${ }^{6}$ V. N. Golovach, A. Khaetskii, and D. Loss, Phys. Rev. Lett. 93, 016601 (2004).

${ }^{7}$ L. M. Woods, T. L. Reinecke, and R. Kotlyar, Phys. Rev. B 69, 125330 (2004).

${ }^{8}$ D. V. Bulaev and D. Loss, Phys. Rev. Lett. 95, 076805 (2005).

${ }^{9}$ J. M. Elzerman, R. Hanson, L. H. Willems van Beveren, B. Witkamp, L. M. K. Vandersypen, and L. P. Kouwenhoven, Nature 430, 431 (2004).

${ }^{10}$ M. Kroutvar, Y. Ducommun, D. Heiss, M. Bichler, D. Schuh, G. Abstreiter, and J. J. Finley, Nature 432, 81 (2004).

${ }^{11}$ J. Cheng, Y. Wu, X. Xu, D. Sun, D. G. Steel, A. S. Bracker, D. Gammon, W. Yao, and L. J. Sham, Solid State Commun. 140, 381 (2006).

${ }^{12}$ D. Heiss, S. Schaeck, H. Huebl, M. Bichler, G. Abstreiter, J. J. Finley, D. V. Bulaev, and D. Loss, Phys. Rev. B 76, 241306(R) (2007).

${ }^{13}$ B. D. Gerardot, D. Brunner, P. A. Dalgarno, P. Öhberg, S. Seidl, M. Kroner, K. Karrai, N. G. Stoltz, P. M. Petroff, and R. J. Warburton, Nature 451, 441 (2008).

${ }^{14}$ J. R. Petta, A. C. Johnson, J. M. Taylor, E. A. Laird, A. Yacoby, M. D. Lukin, C. M. Marcus, M. P. Hanson, and A. C. Gossard, Science 309, 2180 (2005).

${ }^{15}$ A. Greilich, D. R. Yakovlev, A. Shabaev, Al. L. Efros, I. A. Yugova, R. Oulton, V. Stavarache, D. Reuter, A. Wieck, and M. Bayer, Science 313, 341 (2006).

${ }^{16}$ F. G. G. Hernandez, A. Greilich, F. Brito, M. Wiemann, D. R. Yakovlev, D. Reuter, A. D. Wieck, and M. Bayer, Phys. Rev. B 78, 041303(R) (2008).

${ }^{17}$ D. Brunner, B. D. Gerardot, P. A. Dalgarno, G. Wüst, K. Karrai, N. G. Stoltz, P. M. Petroff, and R. J. Warburton, Science 325, 70 (2009).
${ }^{18}$ I. A. Merkulov, Al. L. Efros, and M. Rosen, Phys. Rev. B 65, 205309 (2002).

${ }^{19}$ S. I. Erlingsson and Y. V. Nazarov, Phys. Rev. B 66, 155327 (2002).

${ }^{20}$ A. V. Khaetskii, D. Loss, and L. Glazman, Phys. Rev. Lett. 88, 186802 (2002)

${ }^{21}$ A. C. Johnson, J. R. Petta, J. M. Taylor, A. Yacoby, M. D. Lukin, C. M. Marcus, M. P. Hanson, and A. C. Gossard, Nature 435, 925 (2005).

${ }^{22}$ P.-F. Braun, X. Marie, L. Lombez, B. Urbaszek, T. Amand, P. Renucci, V. K. Kalevich, K. V. Kavokin, O. Krebs, P. Voisin, and Y. Masumoto, Phys. Rev. Lett. 94, 116601 (2005).

${ }^{23}$ B. Eble, C. Testelin, P. Desfonds, F. Bernardot, A. Balocchi, T. Amand, A. Miard, A. Lemaître, X. Marie, and M. Chamarro, Phys. Rev. Lett. 102, 146601 (2009).

${ }^{24}$ J. Fischer, W. A. Coish, D. V. Bulaev, and D. Loss, Phys. Rev. B 78, 155329 (2008).

${ }^{25}$ C. Testelin, F. Bernardot, B. Eble, and M. Chamarro, Phys. Rev. B 79, 195440 (2009)

${ }^{26}$ P. Desfonds, B. Eble, F. Fras, C. Testelin, F. Bernardot, M. Chamarro, B. Urbaszek, T. Amand, X. Marie, J.-M. Gérard, V. Thierry-Mieg, A. Miard, and A. Lemaître, Appl. Phys. Lett. 96, 172108 (2010)

${ }^{27}$ E. A. Chekhovich, A. B. Krysa, M. S. Skolnick, and A. I. Tartakovskii, Phys. Rev. Lett. 106, 027402 (2011).

${ }^{28}$ P. Fallahi, S. T. Yilmaz, and A. Imamoglu, Phys. Rev. Lett. 105, 257402 (2010)

${ }^{29}$ The time scale of the carrier spin relaxation imposed by nuclear dipole-dipole interaction is of the order of $100 \mu \mathrm{s}$.

${ }^{30}$ M. Yu. Petrov, G. G. Kozlov, I. V. Ignatiev, R. V. Cherbunin, D. R. Yakovlev, and M. Bayer, Phys. Rev. B 80, 125318 (2009).

${ }^{31}$ A. V. Khaetskii, D. Loss, and L. Glazman, Phys. Rev. B 67, 195329 (2003).

${ }^{32}$ G. G. Kozlov, J. Exp. Theor. Phys. 105, 803 (2007).

${ }^{33}$ B. Eble, P. Desfonds, F. Fras, F. Bernardot, C. Testelin, and M. Chamarro, A. Miard and A. Lemaitre, Phys. Rev. B 81, 045322 (2010).

${ }^{34}$ E. Aubry, C. Testelin, F. Bernardot, M. Chamarro, and A. Lemaître, Appl. Phys. Lett. 90, 242113 (2007).

${ }^{35}$ F. Bernardot, E. Aubry, C. Testelin, J. Tribollet, M. Chamarro, L. Lombez, P.-F. Braun, X. Marie, T. Amand, and J. M. Gérard, Phys. Rev. B 73, 085301 (2006).

${ }^{36}$ C. Testelin, B. Eble, F. Bernardot, G. Karczewski, and M. Chamarro, Phys. Rev. B 77, 235306 (2008). 
${ }^{37}$ J. S. Colton, T. A. Kennedy, A. S. Bracker, and D. Gammon, Phys. Rev. B 69, 121307(R) (2004).

${ }^{38}$ M. Ikezawa, B. Pal, Y. Masumoto, I. V. Ignatiev, S. Yu. Verbin, and I. Ya. Gerlovin, Phys. Rev. B 72, 153302 (2005).

${ }^{39}$ P. Maletinsky, A. Badolato, and A. Imamoglu, Phys. Rev. Lett. 99, 056804 (2007).

${ }^{40}$ M. Trif, P. Simon, and D. Loss, Phys. Rev. Lett. 103, 106601 (2009).

${ }^{41}$ Hai Wei, Ming Gong, G-C Guo, and Lixin He, e-print arXiv:1011.4724, (2010).

${ }^{42}$ K.A. Al-Hassanieh, V.V. Dobrovitski, E. Dagotto and B.N. Harmon, Phys. Rev. Lett. 97, 037204 (2006)

${ }^{43}$ B. Pal, S. Yu. Verbin, I. V. Ignatiev, M. Ikezawa, and Y. Masumoto, Phys. Rev. B 75, 125322 (2007).

${ }^{44}$ C. Emary, X. Xu, D. G. Steel, S. Saikin, and L. J. Sham, Phys. Rev. Lett. 98, 047401 (2007).
${ }^{45}$ X. Xu, Y. Wu, B. Sun, Q. Huang, J. Cheng, D. G. Steel, A. S. Bracker, D. Gammon, C. Emary, and L. J. Sham, Phys. Rev. Lett. 99, 097401 (2007).

${ }^{46}$ M. Atature, J. Dreiser, A. Badolato, A. Högele, K. Karrai, and A. Imamoglu, Science 312, 551 (2006).

${ }^{47}$ A. J. Ramsay, S. J. Boyle, R. S. Kolodka, J. B. B. Oliveira, J. Skiba-Szymanska, H. Y. Liu, M. Hopkinson, A. M. Fox, and M. S. Skolnick, Phys. Rev. Lett. 100, 197401 (2008).

${ }^{48}$ A. Shabaev, Al. L. Efros, D. Gammon, and I. A. Merkulov, Phys. Rev. B 68, 201305(R) (2003).

${ }^{49}$ The experimental $T_{N}^{h}$ value is independent of the excitation density. ${ }^{50}$ A. Greilich, R. Oulton, E. A. Zhukov, I. A. Yugova, D. R. Yakovlev, M. Bayer, A. Shabaev, Al. L. Efros, I. A. Merkulov, V. Stavarache, D. Reuter, and A. Wieck, Phys. Rev. Lett. 96, 227401 (2006). 